\title{
SIMULAÇÃO COMPUTACIONAL APLICADA À MODELAGEM DO PROCESSO DE RECEBIMENTO DE UNIFORMES NA MARINHA DO BRASIL
}

\author{
Carlos Ribeiro Praia ${ }^{1}$ \\ Carlos Francisco Simões Gomes ${ }^{2}$
}

\begin{abstract}
RESUMO: A Marinha do Brasil procura fazer que as atividades de apoio estejam sempre alinhadas com os objetivos da instituição, ao avaliar constantemente a possibilidade de emprego das melhores técnicas de gestão nas organizações militares. O propósito deste artigo é procurar uma nova proposta de melhoria em um processo logístico na MB, concentrando esforço no estudo da teoria das filas e da simulação. Para isso, é utilizada simulação computacional no processo de recebimento de uniformes, de modo a auxiliar na identificação de gargalos no fluxo de material do Sistema de Abastecimento da Marinha e no melhor uso dos recursos operacionais necessários à execução das atividades logísticas. O emprego de tais técnicas da pesquisa operacional justifica-se pela capacidade de permitir uma adequada análise dos diferentes cenários e políticas operacionais, bem como, uma correta avaliação de cada ponto crítico do sistema real. Os resultados das simulações mostraram que a diminuição do tempo de uma etapa provocava aumento da fila na etapa seguinte. Concluiu-se, então, que o aumento da capacidade do sistema deveria ser aplicado sempre nos pontos de restrição e repetido até alcançar a capacidade e o tempo de processamento desejado.
\end{abstract}

PALAVRAS CHAVE: simulação computacional, uniformes, Marinha do Brasil.

The Navy of Brazil seeks to support activities are always aligned with the goals of the institution, to constantly evaluate the possibility of using the best techniques of management in military organizations. The purpose of this article is look for a new proposal to improve logistics in a process in $\mathrm{MB}$, concentrating efforts in the study of queuing theory and simulation. For this, computer simulation is used in the process of receiving uniforms in order to assist in identifying bottlenecks in the flow of material from the Navy Supply System and the better use of operational resources needed to implement its logistics activities. The use of such operational research techniques is justified by its ability to allow a proper analysis of different scenarios and operational policies as well, an accurate evaluation of each critical point of the real system. Simulation results showed that decreasing the time step caused an increase in the queue the next step. Thus, it was concluded that the increased capacity of the system should be always applied at the point of restriction and repeated to reach the capacity and the desired processing time.

KEYWORDS: computer simulation, uniform, Brazilian Navy.

1 Universidade Federal Fluminense e Marinha do Brasil praia@den.mar.mil.br

${ }_{2}$ Universidade Federal Fluminense cfsg1@bol.com.br 


\section{INTRODUÇÃO}

A evolução no mundo do trabalho traz hoje novos desafios que substituem os velhos paradigmas, afetando as relações entre as pessoas (Milaré e Yoshida, 2009).

Assim, mudanças significativas estão ocorrendo na gestão de diversas organizações no mundo, reflexo de um novo cenário de globalização da economia. Devido a essas mudanças, as organizações estão sendo obrigadas a reformular seus processos, por meio de eficientes sistemas de gestão, de modo a fazer frente às exigências da concorrência e às necessidades do mercado consumidor. Compreender os processos que permitem garantir a eficiência do fluxo de material possibilita o desenvolvimento de melhorias que podem apresentar resultados mais significativos em termos de preparação e atualização logísticas.

A logística está presente em toda atividade empresarial moderna, os impactos dos erros e acertos nesse setor, refletem-se diretamente nos consumidores finais (Menezes et al., 2012).

Com um mercado cada vez mais competitivo e de mudanças rápidas, a simulação tem-se tornado uma ferramenta útil e poderosa à análise de sistemas complexos e à resolução de problemas, indispensáveis aos engenheiros, projetistas e administradores. Ela analisa problemas e serve como ferramenta de análise de sistemas complexos reais ou sob condições específicas determinadas pelo usuário (Pereira e Costa, 2012).

Os ambientes de desenvolvimento de modelos de simulação a eventos discretos podem ser definidos como ferramentas computacionais que auxiliam a análise de problemas, que vão de logística e manufatura até redes de computadores, sem recorrer a modelos analíticos de teoria das filas (Peixoto et al., 2013); (Martins, 2013).

A crescente aplicação da engenharia de processos nas organizações resulta de uma necessidade de maior flexibilidade, coordenação de atividades e orientação para resultados integrados nas organizações. De certa forma, a abordagem por processos se contrapõe à estruturação das organizações funcionais. Por um lado a organização funcional potencializa o aumento de conhecimento especializado na medida em que agrupa os profissionais por especialidade técnica e prioriza os indicadores de desempenho departamentais. Por outro, não garante, necessariamente, um ótimo desempenho global da organização e, em alguns casos, pode estimular um comportamento de isolamento e de competitividade interna (Lacerda, Rodrigues e Silva, 2011).

Para a implementação das mudanças relativas à melhoria dos processos é necessário tomar decisões. Desse modo, constitui-se um desafio à tarefa de decidir aquelas que melhor atendam aos objetivos do sistema e que proporcionem aos tomadores de decisão a certeza de que estão utilizando as melhores técnicas de gestão.

As ferramentas fornecidas pela Pesquisa Operacional (PO) oferecem a mesma solução que um decisor poderia chegar por meio da intuição. Todavia, os benefícios gerados pela utilização da PO devem ser avaliados considerando seu impacto em longo prazo no processo gerencial. Ademais, essas ferramentas podem ser abordadas por meio de modelos determinísticos de programação matemática, ou através de modelos probabilísticos que consideram as incertezas dos modelos, como teoria das filas e simulação.

A partir desse contexto, a Marinha do Brasil (MB) busca implementar soluções alternativas que visam garantir sua eficiência logística. Entretanto, sem uma visão do impacto de uma mudança, a MB ficaria exposta a altos riscos de dano a seu patrimônio e a sua imagem. A simulação pode ser empregada no auxílio ao processo decisório, levando-se em conta um cenário extraído do mundo real, onde as possíveis alternativas são consideradas como variáveis em um modelo matemático que expressa o cenário analisado.

Não se observa especificamente uma abordagem que auxilie os gestores a tornar operacionais os conceitos relacionados à teoria das filas e simulação em processos de recebimento de material em organizações. Ainda assim, existe uma infinidade de trabalhos, dentre os quais é possível citar alguns envolvendo simulação em setores 
públicos, saúde e logística, que nortearam esta pesquisa.

Leal (2003) apresentou um estudo para diagnosticar o processo de atendimentos a clientes em um banco, por meio do emprego de técnicas de mapeamento de processos e simulação computacional.

Valença (2011) propôs um estudo de simulação na área da saúde com objetivo de analisar o fluxo de usuários em uma policlínica da $\mathrm{MB}$ e de avaliar e dimensionar os recursos humanos e a estrutura de atendimento prestado.

Por sua vez, Pastore, Guimarães e Diallo (2010) demonstraram a aplicabilidade da simulação computacional no setor logístico ao realizar um estudo de modelagem das atividades de atendimento de pedidos de uniformes da MB.

Tal qual o trabalho anterior, vislumbra-se com este propor soluções que elevem o nível de eficiência e eficácia das atividades do outro elo da cadeia logística.

\subsection{Propósito do artigo}

Este artigo tem como propósito procurar uma nova proposta de melhoria em um processo logístico, cujo enfoque consiste na simulação computacional de sistema dinâmico de eventos discretos, envolvendo a análise de um exemplo de sistema de fila de canal simples e multifásico, por meio da modelagem do processo de recebimento de uniformes da MB.

\section{REVISÃO DA LITERATURA}

\subsection{Teoria das filas}

Segundo Hillier e Lieberman (2007), a teoria das filas consiste no estudo da espera em todas as formas diversas de filas cujos modelos são usados para representar os diversos tipos de sistemas de filas que surgem na prática. De acordo com Moreira (2007), as pessoas associam a existência de filas a um excesso de demanda de um serviço sobre a capacidade de atendimento. Ou seja, há mais elementos a atender do que postos de serviço para atendimento. Isso é uma verdade, mas não é toda. Às vezes, a capacidade de atendimento é o bastante, mas a própria dinâmica do sistema leva à formação de filas. Na sua maioria, é impossível programar as filas, pois as chegadas e os serviços são geralmente aleatórios.

As filas precisam ser gerenciadas, ou seja, alguém tem de mantê-las sob controle. A situação mais ideal é um sistema de fila balanceado que tende a um equilíbrio. Assim, existem três tipos de análise de sistemas de fila: tentativa e erro, analítico e métodos de simulação. Este estudo usará a simulação.

Um determinado projeto pode evoluir na base de tentativa e erro para um sistema simples. Entretanto, para sistema complexo, esse método é inapropriado. A outra abordagem está relacionada ao emprego do método analítico, onde são desenvolvidos modelos matemáticos e estatísticos que descrevem o comportamento do sistema. Essa abordagem deve ser usada sempre que possível, sendo que vários sistemas de filas são muito complexos para serem estudados através desse método. Por sua vez, o tempo requerido para a resolução de problemas complexos foi reduzido de modo considerável, ao se utilizar a simulação. O tempo de operação ficou mais curto e o de programação diminuiu com o uso de linguagens e de softwares de simulação.

\subsection{Simulação}

A simulação a eventos discretos (ou simulação discreta) teve grande expansão nas últimas quatro décadas, ganhando contorno científico bem definido em congressos e periódicos especializados no assunto (Rangel et al., 2012).

A simulação tem sido uma técnica utilizada para auxiliar processos de tomada de decisão, abrangendo decisões de investimento em infraestrutura e na melhoria de operações, entre outras. Além de reduzir prejuízos causados por investimentos contestáveis, essa técnica da PO permite também reduzir o tempo de observação dos efeitos de decisões, visto que, com o auxílio do computador, pode-se simular em alguns minutos $\mathrm{O}$ comportamento de um processo ou sistema real. Muitos trabalhos da literatura já estudaram a importância da simulação como aliada em gerência de operações (Cardoso, Raupp e Diallo, 2012).

Segundo Silva Junior et al. (2011), 
inserida no campo de conhecimento da PO, a simulação é uma representação da operação de um processo ou sistema real, num dado período de tempo, que envolve a geração de uma história artificial desse sistema e a observação desta para fazer inferências relativas às características do processo real. A simulação possui dependência com o tipo de variável a ser considerada no modelo. A simulação de eventos discretos abrange o estudo de modelos de simulação cujas variáveis mudam de estado instantaneamente em pontos específicos de tempo, em contraste ao que ocorre com modelos contínuos, cujas variáveis podem mudar de estado continuamente no decorrer do tempo.

De forma geral, a simulação e a modelagem podem ser consideradas técnicas integradas, sendo necessário o desenvolvimento de um modelo para posteriormente simulá-lo. Normalmente, os modelos incorporam várias entradas (parâmetros) no sistema, como tempo, distância, velocidade e recursos disponíveis, e fornecem um meio estatisticamente válido para avaliar, redesenhar e quantificar a utilização de recursos, a racionalização de processos e o tempo gasto (Pereira e Costa, 2012).

A simulação é uma técnica muito utilizada para projetar um modelo de um sistema real ou proposto e realização de experiências com este modelo. Frequentemente, a finalidade das experiências é a estimar os efeitos sobre o desempenho do sistema devido a alterações em um conjunto de variáveis de entrada controláveis. No entanto, o modelo de simulação pode ser bastante complexo (Santos e Santos, 2009); (Poropudas e Virtanen, 2011).

Um analista de simulação tem, normalmente, quatro alternativas, quando deseja construir um modelo de simulação a eventos discretos. A primeira é trabalhar com uma linguagem de uso geral somente, a segunda é trabalhar com as bibliotecas de simulação, a terceira alternativa é trabalhar com linguagens específicas para simulação e, por último, trabalhar com os ambientes de simulação a eventos discretos (Peixoto $e t$ al., 2013).

\section{FORMULAÇÃO DO PROBLEMA}

A Base de Abastecimento da Marinha no Rio de Janeiro (BAMRJ) é um complexo logístico, cuja finalidade principal é consolidar, em uma mesma área, depósitos centrais que funcionam como Centros de Distribuição (CD) de materiais utilizados nas rotinas administrativas e operacionais da MB. Dentre os diversos CD, o Depósito de Fardamento da Marinha no Rio de Janeiro (DepFMRJ) tem papel de significativa importância no Sistema de Abastecimento da Marinha (SAbM), uma vez que movimenta e manuseia itens essenciais na logística da Instituição.

Segundo Brasil (2009), cabe ao SAbM o exercício da atividade de abastecimento na MB, sendo constituído de órgãos, processos e recursos com a finalidade de promover, manter e controlar o provimento do material necessário à manutenção das organizações em condição de plena eficiência. Assim, dentro do escopo do estudo, a sistemática geral de uniformes na MB está estruturada da seguinte maneira:

- Centro de Controle de Inventário da Marinha (CCIM): é o Órgão de Controle responsável pelo controle dos níveis de estoque e por ações de recompletamento, redistribuição e destinação de excessos.

- Centro de Obtenção da Marinha no Rio de Janeiro (COMRJ): é o Órgão de Obtenção no país responsável pela aquisição do material.

- Depósito de Fardamento da Marinha no Rio de Janeiro (DepFMRJ): é o Órgão de Distribuição responsável pelo recebimento, armazenagem e expedição de todos os itens de fardamento que fazem parte de linha de fornecimento do SAbM.

- Posto de Distribuição e de Encomenda de Uniformes (PDU/PEU): é o centro de acumulação de itens de fardamento. É o último link entre o usuário (militar) e o SAbM.

A Figura 1 ilustra a movimentação do material, desde o fornecedor até $\mathrm{o}$ usuário final. 


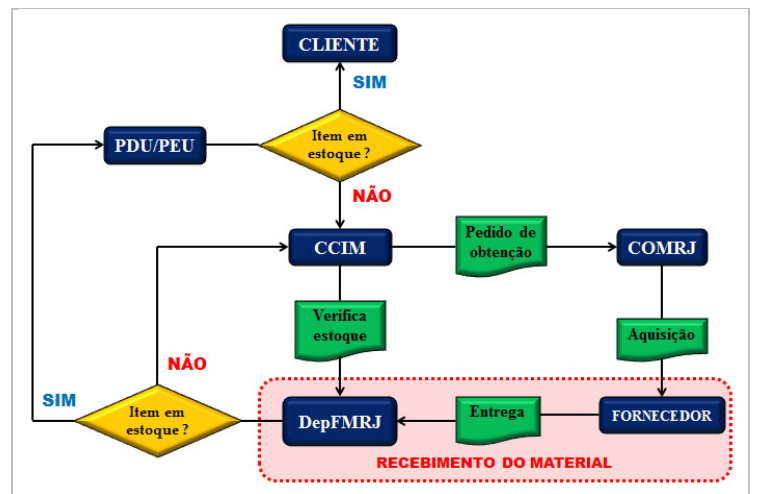

Figura 1 - Sistemática geral de uniformes na MB

Para Pastore, Guimarães e Diallo (2010), a motivação pela procura por novas soluções de otimização das operações pauta-se na premissa de se buscar um nível ótimo de atendimento, ao menor custo possível. Para a MB, as atividades logísticas têm importância estratégica, uma vez que são vitais na manutenção de seu estado de prontidão e de aprestamento para a defesa nacional.

Assim, a finalidade do trabalho foi de contribuir para o aumento da eficiência das operações logísticas do DepFMRJ, considerando como ponto de partida do modelo a data de entrega do material pelo fornecedor e, como término, a data da efetiva arrecadação do item no Sistema de Informações Gerenciais do Abastecimento (SINGRA).

Para a modelagem, foram consideradas todas as etapas que compõem o processo, sendo este representado na Figura 2.

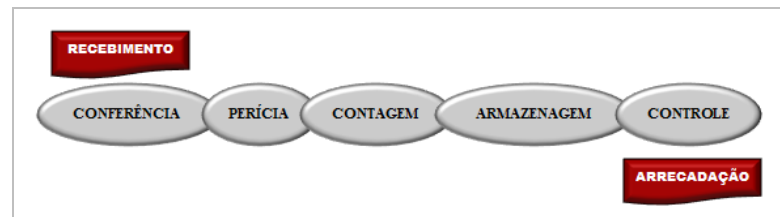

Figura 2 - Fluxo do processo de recebimento de uniformes

O estudo consistiu em criar um modelo que daria argumentos para possíveis adoções de estratégias que melhorassem os resultados do sistema, tornando o processo mais eficiente e eficaz no que diz respeito ao recebimento de material. Para isso, foram verificados os tempos despendidos para realização das etapas de conferência, perícia, contagem, armazenagem e controle.

Foi apresentado, ainda, o ponto crítico na logística interna do depósito, bem como proposta para solucioná-lo, a qual resultou em uma metodologia de modelagem com emprego de simulação computacional e sugestões de mudança de alguns procedimentos internos que contribuíram para a formação dos gargalos.

Os objetivos foram os seguintes:

\section{Objetivos gerais}

- Construir um modelo de sequenciamento do recebimento do material, com o propósito de contribuir para análise de desempenho do processo.

- Implementar a modelagem desenvolvida no software de simulação.

\section{Objetivos específicos}

- Verificar a duração do ciclo das etapas envolvidas no processo de recebimento.

- Analisar o procedimento operacional empregado, levandose em consideração a capacidade de recursos do depósito.

\section{METODOLOGIA DA PESQUISA}

Para Silva e Menezes (2001), a metodologia de pesquisa é entendida como um conjunto de etapas ordenadamente 
dispostas que devem ser vencidas na investigação de um fenômeno. Inclui a escolha do tema, o planejamento da investigação, desenvolvimento metodológico, a coleta e a tabulação de dados, a análise de resultados. Os tipos de pesquisa apresentados nas diversas classificações não são estanques. Uma mesma pesquisa pode estar enquadrada em várias classificações, desde que obedeça aos requisitos inerentes a cada tipo.

De acordo com as classificações apresentadas por Gil (2007), esta pesquisa é do tipo aplicada, por envolver aplicações práticas no recebimento de material; e exploratória, por proporcionar maior familiaridade com o problema, tornando-o mais explícito. A análise de dados apresenta características quantitativas, com descrição de procedimentos e análise de resultados numéricos.

Quanto ao procedimento empregado, trata-se de um estudo de caso que foi desenvolvimento, por meio das etapas descritas a seguir:

a) Inicialmente, foi determinado $\mathrm{o}$ problema que seria a base para o estudo, seguindo-se uma revisão bibliográfica sobre os assuntos referentes ao trabalho.

b) Posteriormente, foi definido o setor em que se desenvolveria o estudo. Nesse caso, o depósito de fardamento.

c) Após, foi determinado qual seria o objeto que faria parte do estudo, uma vez que se pretendia ampliar os conhecimentos acerca de uma categoria de material específica.

d) Foi elaborado um roteiro para o trabalho, contendo procedimentos, objetivos e cronograma.

e) Em seguida, foi realizado o levantamento de dados e a coleta das informações.

f) Após a coleta dos dados e análise dos mesmos, foi verificada como a situação poderia ser explorada, segundo a bibliografia pesquisada na etapa anterior. Nesta etapa, também, foi analisada a proposição do modelo e seus resultados, considerando as características qualitativas e quantitativas que envolveram o levantamento de dados e a descrição dos procedimentos.

g) Por fim, foram apresentadas as conclusões com base nas informações obtidas nas etapas anteriores.

\section{DESENVOLVIMENTO DAS SIMULAÇÕES}

A simulação pode ser aplicada para várias situações dentro do campo da logística, por meio da avaliação da interação entre os componentes que integram o sistema. É utilizada, por exemplo, para análise de linhas de fabricação e montagem, de sistemas de armazenagem e até mesmo de movimentação de materiais.

Medição e controle de tempo em cadeias produtivas e logísticas podem ser úteis na formulação e execução de estratégias; e um modo de gerenciar o tempo como recurso produtivo é medir e controlar estes tempos (Sellitto e Walter, 2008).

Como mencionado anteriormente, este trabalho consistiu de um estudo de caso, cujo objetivo principal foi desenvolver e implementar um modelo de um processo específico do fluxo logístico do SAbM, de modo a identificar gargalos no mesmo. Foi escolhido o processo de recebimento uniformes do DepFMRJ para análise através da simulação.

Os passos utilizados para seu desenvolvimento foram os mesmos sugeridos por Law e Kelton (2000):

i. Formulação do problema e planejamento do estudo;

ii. Coleta de dados e definição do modelo;

iii. Validação do modelo conceitual;

iv. Construção do programa computacional e verificação;

v. Realização de execuções piloto;

vi. Validação do modelo programado;

vii. Projeto dos experimentos;

viii. Realização das execuções de simulação;

ix. Análise de resultados; e

x. Documentação e implementação.

A utilização da técnica de simulação para este estudo pode ser justificada pelos 
benefícios de economia de tempo e de recursos, sejam eles físicos ou financeiros. Além disso, pode-se afirmar que a modelagem é facilmente entendida em virtude da impressão que se tem do real controle e gerenciamento do modelo.

Todo o desenvolvimento do modelo foi realizado na versão student do software Arena $^{\circledR}$ (versão 14.0). O estudo apoiou-se, basicamente, de alterações nos recursos, parâmetros, funções e interações do sistema, com a finalidade de se verificar os efeitos dessas variações no modelo.

Segundo Prado (2010), o Arena ${ }^{\circledR}$ é representado por um ambiente gráfico integrado de simulação, que contém todos os recursos para modelagem de processos, desenho e animação, análise estatística e análise de resultados.

Inicialmente, foi definido o sistema que seria abordado no presente trabalho. $\mathrm{O}$ modelo para as simulações referiu-se a um sistema de fila, referente ao processo de recebimento de material do depósito de fardamento. Esse processo foi escolhido em virtude do alto tempo envolvido em sua operação, sendo esta constituída pelo período desde a entrega do item pelo fornecedor até sua disponibilização no SINGRA. Trata-se, portanto, de um exemplo de problema de fila inerente ao escopo do estudo e bastante comum em diversas organizações. Foram realizadas algumas suposições sobre modelo:

a) $\mathrm{O}$ intervalo de tempo entre as chegadas do material foi considerado um valor estocástico com distribuição de probabilidade conhecida, o que permitiu que $\mathrm{o}$ modelo fosse mais fiel à realidade;

b) A probabilidade de uma chegada durante um intervalo de tempo específico permaneceu constante $\mathrm{e}$ independente do número de chegadas anteriores e do tempo de espera;

c) Antes de estar disponível no SINGRA, o material recebido teve que passar necessariamente pelas cinco etapas do processo: Conferência, Perícia, Contagem, Armazenagem e Controle; e

d) O tempo gasto pelo material em cada etapa também foi considerado um valor estocástico com distribuição de probabilidade conhecida.
O modelo foi desenvolvido conforme o sistema real existente, sendo constituído por cinco estações de processamento. $\mathrm{Na}$ primeira estação, realiza-se a conferência do material, por meio da verificação da conformidade do item que está sendo recebido com o documento emitido pelo COMRJ e pelo fornecedor. $\mathrm{Na}$ segunda estação, uma amostra do item é conduzida para perícia, a fim de se certificar da adequação do material à norma técnica. $\mathrm{Na}$ terceira estação, é realizada a contagem do material recebido. Na quarta estação, o item é armazenado conforme localização indicada pelo SINGRA. Por fim, a quinta estação finaliza todo o processo de arrecadação, passando, assim, o item a estar disponível para fornecimento. Uma vez que o tempo de processo varia, filas de itens a serem processados se formam em frente às estações de processamento.

Para Harrel, Ghosh e Bowden (2004) a coleta de dados é um dos pontos mais importantes do processo de simulação, uma vez que, se os mesmos não forem consistentes, o modelo também não o será. Assim, dados referentes aos recebimentos dos itens durante os meses de janeiro a maio de 2013 foram coletados de modo a calcular o intervalo entre as chegadas dos itens.

O tempo calculado seguiu uma curva definida no Arena Input Analyzer. Apesar de a distribuição Beta ter sido a indicada pela ferramenta, optou-se pela distribuição Exponencial, por esta melhor representar o tempo decorrido entre dois eventos. É possível observar que, na Tabela 1 , os valores do erro quadrático estão muito próximos nas três primeiras curvas, o que permite afirmar que qualquer uma delas, com seus parâmetros, produz praticamente o mesmo efeito. Além disso, o valor de $\mathrm{p}=$ 0,721 foi grande o suficiente para se confiar nos resultados do ajuste. Conforme os dados observados, a expressão foi representada por $7.5+$ EXPO (2.55), com erro de 0,00434 . 
Tabela 1 - Valores do erro para cada distribuição

\begin{tabular}{l|c}
\multicolumn{1}{c|}{ Função } & Erro quadrático \\
\hline Beta & 0,00367 \\
\hline Erlang & 0,00434 \\
\hline Exponencial & 0,00434 \\
\hline Poisson & 0,00937 \\
\hline Lognormal & 0,01100 \\
\hline Weibull & 0,01100 \\
\hline Gama & 0,01280 \\
\hline Triangular & 0,04560 \\
\hline Normal & 0,07050 \\
\hline Uniforme & 0,08760 \\
\hline
\end{tabular}

A parte de planejamento de estudo consistiu na definição de alguns outros aspectos, como configurações do sistema modelado, objetivos do estudo e medidas de desempenho.

Alguns termos referentes à teoria das filas foram correlacionados com os termos do sistema simulado:

a) Entidades (clientes): corresponderam aos itens do sistema. Apesar de os dados coletados terem se referido aos itens camisa branca, cinza e azul. Para o modelo, existiu apenas um tipo, visto que é empregado o mesmo procedimento no recebimento dos três itens.

b) Capacidade da fila (linha de espera): referiu-se ao número admissível de itens na fila. No caso do modelo, a capacidade foi infinita.

c) Canal de atendimento: canal simples com cinco estações de processamento.

d) Capacidade dos servidores: referiu-se ao número de clientes sendo atendidos ao mesmo tempo. Para todas as estações, foi utilizada a capacidade de um item.

e) Estratégias de admissão: todos os itens foram admitidos no sistema.

f) Chegada dos clientes: considerou-se que os itens chegavam de acordo com uma distribuição de Poisson. Essa distribuição corresponde aos sistemas de filas em que a chegada de uma entidade independe da chegada da outra.

g) Prioridade da fila: diz respeito ao método de decisão de atendimento do cliente. No modelo, o item que chegou primeiro foi atendido primeiro.

h) Tamanho da população: para o modelo a população foi considerada infinita.

Um aspecto importante considerado foi que, por ocasião da entrada do item no sistema, os recursos de pessoal e de material estiveram sempre disponíveis. Por outro lado, outros aspectos da operação real foram desconsiderados. Por exemplo, não se diferenciou o tempo de preparação do tempo em que efetivamente a atividade foi executada. Esse e outros detalhes foram considerados desnecessários para $\mathrm{o}$ desenvolvimento do modelo deste estudo.

Para inserção das informações referentes ao tempo despendido em cada etapa do processo, dados foram coletados no DepFMRJ, a fim de se calcular o tempo médio de processamento de cada estação do modelo, sendo este representado pelas curvas de probabilidade obtidas pelo Arena Input Analyzer e apresentadas na Tabela 2. Os tempos foram expressos em dias.

Tabela 2 - Curvas do modelo inicial

\begin{tabular}{l|c|c}
\hline \multicolumn{1}{c|}{ Processo } & Distribuição de probabilidade & Erro quadrático \\
\hline Conferência & $2.5+9 *$ BETA $(0.528,1.24)$ & 0.009718 \\
\hline Perícia & $5.5+13 *$ BETA $(0.527,1.19)$ & 0.004434 \\
\hline Contagem & $2.5+9 *$ BETA $(0.437,0.949)$ & 0.006112 \\
\hline Armazenagem & $1.5+6 *$ BETA $(0.632,1.57)$ & 0.006241 \\
\hline Controle & $0.5+7 *$ BETA $(0.654,1.3)$ & 0.009040 \\
\hline
\end{tabular}

Nos testes de aderência realizados, para cada curva testada foi calculado um valor para o erro quadrático. Após realizar este cálculo para todas as possíveis distribuições de probabilidade, o Input Analyzer ordenou as curvas conforme esse valor. A função Beta foi escolhida por apresentar o menor erro. 
Não houve maiores problemas para que se adotassem as expressões apontadas, visto que os dados coletados foram bastante consistentes e representativos do ambiente modelado.

Apesar de o modelo poder ser ampliado para incluir, por exemplo, múltiplos itens, este ficou restrito à simulação de filas de canal simples multifásico de um único item, tendo como argumento sua simplificação.

O sistema foi estudado a partir da redução dos tempos de processamento observados inicialmente. As novas distribuições de probabilidade referiram-se à redução pela metade dos valores do tempo de processamento das estações, simulando o aumento da produtividade do pessoal de cada estação. As novas distribuições constam na Tabela 3.

Tabela 3 - Curvas resultantes da redução no tempo de processamento

\begin{tabular}{l|c|c}
\hline \multicolumn{1}{c|}{ Processo } & Distribuição de probabilidade & Erro quadrático \\
\hline Conferência & $0.09+4.82 *$ BETA $(0.703,1.56)$ & 0.015800 \\
\hline Perícia & $5.2+3.61 *$ BETA $(0.731,1.43)$ & 0.009545 \\
\hline Contagem & $1.1+4.8 *$ BETA $(0.578,1.19)$ & 0.010100 \\
\hline Armazenagem & $0.09+4.82 *$ BETA $(0.592,1.23)$ & 0.007674 \\
\hline Controle & $0.19+3.61 *$ BETA $(0.741,1.43)$ & 0.009845 \\
\hline
\end{tabular}

Tal redução de tempo poderia ser conseguida através da eliminação de redundâncias, uma vez que foi verificado um controle excessivo do processo por meio de registros em planilhas eletrônicas; da revisão do procedimento padrão empregado pelo pessoal envolvido nas diversas etapas, com vistas a eliminar as tarefas desnecessárias; do emprego de melhores recursos operacionais; e, até mesmo, da melhoria do sistema de gerenciamento empregado (SINGRA). Para a escolha das distribuições da Tabela 3, foi adotado o mesmo procedimento para as curvas obtidas na Tabela 2, alterando-se os valores das amostras.

A partir da combinação dessas novas distribuições com as anteriores, foram obtidos trinta e dois modelos, compreendendo desde a alteração apenas do tempo da estação CONFERỂNCIA até de todas elas simultaneamente.

Para avaliação da eficácia das diferentes configurações do sistema, foram utilizadas algumas medidas de desempenho:

a) Tempo médio no sistema: tempo médio desde a chegada do item (CONFERÊNCIA) até o término do seu processamento (CONTROLE).

b) Tamanho máximo das filas: quantidade de itens em espera para processamento. c) Média de itens processados: quantidade média de itens atendidos por todas as estações.

d) Eficiência do sistema: relação entre a média de itens que chegaram e a média de itens processados no período equivalente há 360 dias. Essa medida visa identificar a quantidade de itens que permanece no sistema como não processado, após esse período.

A validação do modelo foi realizada através de sua análise detalhada, bem como por meio de consultas a alguns setores do DepFMRJ. O objetivo foi garantir a veracidade das suposições do modelo, tornando menor a probabilidade de erros.

A figura 3 mostra a lógica do modelo final. Note-se que nele constam as cinco etapas correspondentes ao sistema real: Seção de Recebimento para conferência, Seção de Perícia, Seção de Recebimento para contagem, Seção de Armazenagem e Seção de Controle. No módulo INÍCIO DO PROCESSO, simulou-se a entrega do item no depósito.

Nos módulos Conferência, Perícia, Contagem e Armazenagem estão as estações de processamento, assim como, suas respectivas estações de trabalho que servem para informar que a entidade ocupou uma estação. . 


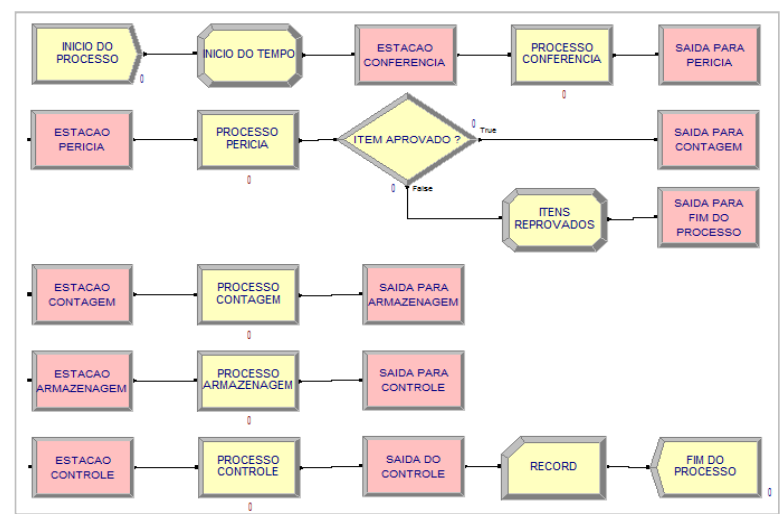

Figura 3 - Ilustração lógica do modelo

Diversas experimentações foram realizadas no modelo de simulação, com o objetivo de fornecer dados para a validação do modelo programado.

A validação do modelo foi realizada pela comparação dos resultados obtidos na simulação do modelo com os dados coletados do sistema real. Além disso, foram realizadas consultas aos operadores do sistema, a fim de se coletar informações acerca da operação em si.

Foi definido o número de simulações que seriam realizadas, a partir da variação dos parâmetros de cada estação. A combinação desses fatores resultou em trinta e dois modelos. Além disso, determinou-se como condição inicial da simulação um sistema de fila totalmente vazio, sem nenhum item.

À medida que as simulações foram executadas, foi possível observar o comportamento das principais variáveis do sistema. Trinta e duas simulações foram executadas, cada uma apresentando uma configuração diferente para o parâmetro "tempo de processamento".
Uma vez que a ideia central do estudo consistiu na verificação da importância do uso da técnica de simulação na identificação de gargalos, para fins de simplificação, decidiu-se mapear o processo, considerando a evolução do item com a ocorrência de casos de discrepância apenas por ocasião da análise pericial do material, visto que os dados coletados no depósito indicavam grande incidência de reprovaçõos nessa etapa. Cerca de $30 \%$ das amostras coletadas eram reprovadas, índice bastante significativo se comparado, por exemplo, à etapa de contagem cujo valor não atingia a $1 \%$.

$\mathrm{O}$ modelo desenvolvido procurou garantir um nível de complexidade apropriado ao estudo e corresponder à realidade.

Para o cálculo do número de replicações, o tempo médio que o item permaneceu no sistema foi considerado como a medida de desempenho mais adequada ao modelo. A Figura 4 exibe o resultado do cálculo do intervalo de confiança realizado no Arena Output Analyzer.

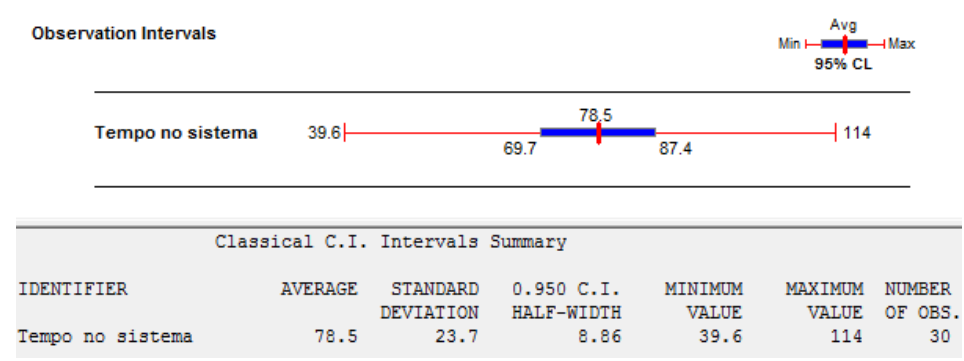

Figura 4 - Intervalo de confiança para 30 replicações Considerando como confiança intervalo de confiança, dado por $h$, foi de estatística 95\%, inicialmente, fez-se a 8,86. Uma vez que se objetivava buscar um experimentação com 30 replicações, onde intervalo em que o valor de $h$ representasse, foi verificada uma média de 78,5 dias e no máximo, $10 \%$ da média amostral, 
realizou-se novo cálculo para determinar o número ideal de replicações. A estimativa para que se alcançasse a meta de $h$ foi de $n^{*}$ igual a 38, conforme pode ser observado na Figura 5.

$$
\begin{aligned}
& \text { Observation Intervals }
\end{aligned}
$$

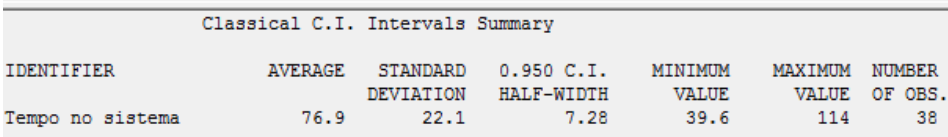

Figura 5 - Intervalo de confiança para 38 replicações

Para validar o modelo, verificou-se se que os tempos estavam coerentes. A estatisticamente a média referente ao tempo Figura 6 mostra uma comparação entre os em cada etapa do processo para os dois valores coletados e os valores gerados na sistemas (real e computacional). Constatou- simulação.

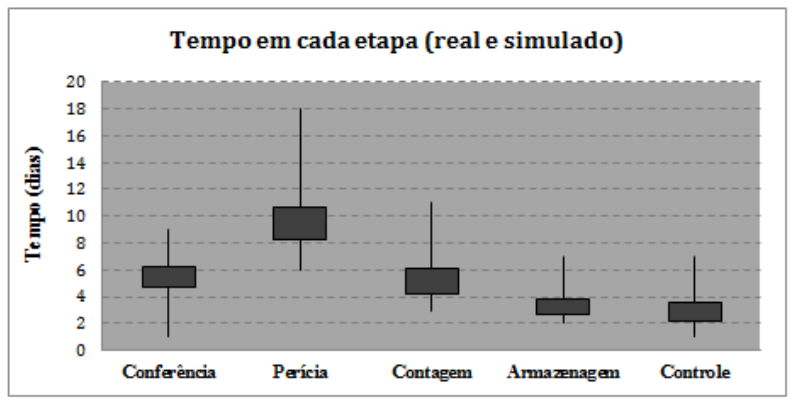

Figura 6 - Comparação dos tempos em cada etapa, real e simulado

As linhas verticais indicam o intervalo dos tempos nas etapas, durante os meses de coleta. A largura das barras horizontais indica a variação dos tempos médios apresentados pela simulação. As barras escuras indicam que o valor apresentado pela simulação está acima do valor médio dos tempos coletados. Foram considerados válidos os casos onde os valores simulados ficaram no intervalo dos valores coletados.

O aumento da capacidade de processamento do sistema poderia ser testado de duas maneiras: a primeira seria aumentando-se o número de estações de processamento da etapa e a segunda diminuindo-se o tempo de processamento da estação, uma vez que, com tal redução, a estação poderia processar uma quantidade maior de elementos dentro do mesmo intervalo de tempo. Tal diminuição seria alcançada por meio da adoção das medidas comentadas anteriormente. Partiu-se do princípio que ambas as soluções seriam equivalentes, sendo, portanto, adotada a segunda alternativa.

A Tabela 4 apresenta os resultados mais significativos das simulações realizadas.

Tabela 4 - Resultados das simulações

\begin{tabular}{|c|c|c|c|c|c|c|c|c|}
\hline \multirow[t]{2}{*}{ Modelo } & \multirow{2}{*}{$\begin{array}{c}\text { Tempo } \\
\text { médio } \\
\text { no } \\
\text { sistema }\end{array}$} & \multicolumn{5}{|c|}{$\begin{array}{c}\text { Tamanho máximo registrado } \\
\text { nas filas }\end{array}$} & \multirow{2}{*}{$\begin{array}{l}\text { Média de } \\
\text { itens } \\
\text { processados }\end{array}$} & \multirow{2}{*}{$\begin{array}{c}\text { Eficiência } \\
\text { do sistema } \\
(\%)\end{array}$} \\
\hline & & C1 & $\mathbf{P}$ & $\mathrm{C} 2$ & $\mathbf{A}$ & C3 & & \\
\hline 1 & 76,9 & 4 & 18 & 2 & 1 & 2 & 24 & 63,2 \\
\hline 2 & 75,2 & 4 & 18 & 2 & 1 & 1 & 24 & 64,8 \\
\hline 3 & 74,7 & 4 & 18 & 2 & 1 & 2 & 24 & 65,2 \\
\hline 5 & 74,5 & 3 & 17 & 0 & 1 & 1 & 24 & 65,2 \\
\hline 9 & 40,9 & 7 & 5 & 5 & 1 & 2 & 32 & 84,2 \\
\hline 11 & 40,7 & 4 & 8 & 2 & 1 & 2 & 32 & 84,2 \\
\hline 16 & 37,2 & 6 & 5 & 1 & 1 & 1 & 33 & 86,8 \\
\hline 17 & 70,5 & 1 & 20 & 1 & 1 & 1 & 25 & 67,6 \\
\hline 28 & 33,7 & 1 & 8 & 2 & 1 & 1 & 34 & 90,2 \\
\hline 32 & 26,2 & 1 & 8 & 1 & 1 & 1 & 35 & 94,1 \\
\hline
\end{tabular}


Legenda: C1 - Conferência; P - Perícia; C2 - Contagem; A - Armazenagem; e C3 - Controle

\begin{tabular}{|c|c|c|c|c|c|}
\hline \multirow[t]{2}{*}{ Modelo } & \multicolumn{5}{|c|}{$\begin{array}{l}\text { Tempo de processamento das } \\
\text { estações (conforme curvas de } \\
\text { distribuição das Tabelas } 2 \text { e } 3 \text { ) }\end{array}$} \\
\hline & C1 & $\mathbf{P}$ & $\mathrm{C2}$ & $\mathbf{A}$ & C3 \\
\hline 1 & 1 & 1 & 1 & 1 & 1 \\
\hline 2 & 1 & 1 & 1 & 1 & 2 \\
\hline 3 & 1 & 1 & 1 & 2 & 1 \\
\hline 5 & 1 & 1 & 2 & 1 & 1 \\
\hline 9 & 1 & 2 & 1 & 1 & 1 \\
\hline 11 & 1 & 2 & 1 & 2 & 1 \\
\hline 16 & 1 & 2 & 2 & 2 & 2 \\
\hline 17 & 2 & 1 & 1 & 1 & 1 \\
\hline 28 & 2 & 2 & 1 & 2 & 2 \\
\hline 32 & 2 & 2 & 2 & 2 & 2 \\
\hline
\end{tabular}

A Tabela 5 indica, para cada modelo usado nos resultados das simulações (Tabela 3), se o tempo de processamento de cada estação usou uma distribuição da Tabela 2 ou 3.

O MODELO 1 representa a situação inicial do sistema. Observa-se que o aumento da capacidade de atendimento surtiu mais efeito quando este foi aplicado aos pontos de gargalo. Entretanto, quando o tempo de processamento foi reduzido apenas nesta etapa, houve uma transferência do gargalo a outro ponto do sistema. Ainda assim, mesmo com a restrição, pôde-se notar um aumento da média de 24 para 32 itens processados. Foi o caso do MODELO 9.

Nas situações em que o tempo de processamento das estações, que não eram gargalos, foi alterado, duas situações foram observadas. Na primeira situação, quando o tempo foi reduzido em uma etapa posterior ao gargalo, não houve aumento da quantidade de itens que chegavam nessa etapa. A eficiência do sistema permaneceu praticamente a mesma (MODELOS 2, 3 e 5 ), caracterizando alta ociosidade e custo desnecessário caso houvesse investimento nessas etapas. Na segunda situação, quando o tempo foi reduzido em uma etapa anterior a do ponto de estrangulamento, situação ficou ainda pior. $\mathrm{O}$ gargalo permanecia agindo como um limitador. A estação que antes já estava sobrecarregada, agora teve que processar um número ainda maior de itens enviados pela etapa anterior. A fila cresceu bastante. Foi o caso do MODELO 17 em que foi registrado um tamanho máximo de 20 itens.

Quanto ao tempo médio de

processamento, verificou-se que este seria tanto menor quanto melhor fosse $\mathrm{o}$ equilíbrio entre as diferentes etapas do processo. Isso quer dizer que, à medida que cada item fosse saindo da etapa, este deveria encontrar a próxima estação livre que, por sua vez, deveria ter acabado de processar outro item. Tal situação pôde ser vista notadamente nos MODELOS 28 e 32.

Com relação ao tamanho máximo da fila registrado no gargalo, pôde-se verificar que existiu uma forte ligação com a eficiência do sistema. Isso quer dizer que, em determinado momento, uma grande quantidade de entidades chegou ao sistema e, portanto, ocorreu um congestionamento. Esse aumento momentâneo no tempo de processamento dos itens foi absorvido por ocasião do cálculo da média, permanecendo, ainda assim, o valor do pico elevado. Tal fato pôde ser observado nos MODELOS 1, 2, 3, 5 e 17. Ressalta-se que o tamanho máximo das filas foi considerado apenas para fins de registro.

Sobre as observações realizadas, é importante registrar que cada caso possui suas particularidades e que aliar o conhecimento prévio do sistema com o conhecimento adquirido através das simulações pode revelar uma maneira de apresentar a resposta de um problema que antes poderia parecer não ter solução.

Quanto aos dois últimos passos da simulação, pode-se dizer que a documentação referiu-se ao registro dos resultados obtidos e dos modelos criados, por meio do desenvolvimento deste estudo. Com relação à implementação, este trabalho se deu, tão somente, à compreensão de um caso específico da $\mathrm{MB}$, não tendo sido, 
portanto, desenvolvido para tal.

\section{CONCLUSÃO}

A ideia central deste estudo consistiu em analisar as características do comportamento e o entendimento dos efeitos da mudança de um processo específico do $\mathrm{SAbM}$, de modo que pudesse oferecer alternativas de solução para entraves no fluxo logístico. O desafio foi saber se a simulação é uma ferramenta eficiente para o estudo de problema de filas e identificação de gargalos.

Tanto o objetivo principal quanto os objetivos secundários deste trabalho foram atingidos, uma vez que a operação de recebimento de uniformes da $\mathrm{MB}$ foi desenvolvida no software de simulação e aplicada na prática; houve medição e análise dos tempos das etapas envolvidas no processo; e foi realizada a verificação do procedimento operacional empregado e da capacidade dos recursos existentes no DepFMRJ.

Os resultados mostraram que o principal gargalo do sistema foi a etapa correspondente à perícia, pelo fato de esta possuir o maior tempo de processamento. Entretanto, por ocasião da realização das simulações, verificou-se que a redução do tempo nessa etapa provocou aumento da fila na etapa de contagem. Essas constatações também foram verificadas nas etapas seguintes na medida em que se diminuía o tempo da etapa anterior.

Concluiu-se, então, que o aumento da capacidade do sistema deveria ser sempre aplicado aos pontos de restrição, e repetido até que se alcançasse a capacidade e o tempo de processamento desejado, ou até que os limites dos recursos fossem atingidos.

Foi observado, também, algumas vantagens do uso da simulação como o experimento de decisões alternativas no processo do DepFMRJ sem intervenções nas suas operações reais, bem como, a capacidade de a simulação comprimir o tempo, permitindo conhecer rapidamente as consequências de longo prazo das decisões tomadas.

Notou-se, ainda, que a simulação teve que ser utilizada de modo bastante coerente, seguindo-se sistematicamente os passos previstos e analisando cuidadosamente os dados coletados.

O desenvolvimento mostrou-se muito valioso, uma vez que foi adequado à análise de diferentes cenários e políticas operacionais e à formação de conceitos do modelo estudado. Isso pôde ser observado através da maneira como a simulação do processo foi capaz de transformar um problema não estruturado em uma grande fonte de conhecimento.

Cabe ressaltar que, embora existam diversas pesquisas envolvendo melhoria de processos nas organizações, não se observa com frequência na literatura uma abordagem específica dos conceitos relacionados à teoria das filas e simulação em sistemas de organizações militares. Portanto, a contribuição deste trabalho consistiu em disseminar os conhecimentos assimilados com o estudo realizado.

Por fim, levando-se em consideração que o caso aqui apresentado é simples, cabe registrar que as observações e conclusões levantadas respondem por apenas uma parte da análise que poderia se fazer do processo.

\section{SUGESTÕES PARA PESQUISA FUTURAS}

A partir do exposto, sugere-se que outros estudos sejam realizados para melhorar e consolidar a metodologia apresentada, inclusive, respeitando as diversas especificidades, aqui não analisadas. Os seguintes pontos podem ser incluídos para futuros desenvolvimentos:

- Ampliação do modelo com a inclusão de múltiplos itens, de modo a propiciar uma maior aproximação ao sistema real;

- Aumento do grau de complexidade, através da previsão de ocorrência de situações de não conformidade, em todas as etapas, bem como, da inclusão, no modelo, dos agentes externos participantes do processo; e

- Inserção do componente custo nos modelos desenvolvidos, visando à garantia de uma melhor análise dos resultados. 


\section{REFERÊNCIAS}

BRASIL. Marinha do Brasil. 2009. Secretaria Geral da Marinha. Normas para execução do abastecimento: SGM-201. $6^{\mathrm{a}}$ Rev. Brasília.

CARDOSO, Í. F., \& RAUPP. F. M. P., DIALLO, M. 2012. Avaliação dos movimentos aéreos no aeroporto do Galeão. Pesquisa Operacional para o Desenvolvimento v. 4, n. 2. p 200-215.

GIL, A. C. 2007. Como elaborar projetos de pesquisa. São Paulo: Atlas.

HARRELL, C.R.; GHOSH, B.K. E \& BOWDEN, R.2004. Simulation Using ProModel, $\quad 2^{\text {a }}$ edição, McGraw-Hill Professional.

HILLIER, F. S.; \& LIEBERMAN, G. J. 2007. Introdução à pesquisa operacional. São Paulo: Editora da Universidade de São Paulo.

LACERDA, D. P.; RODRIGUES, L. H.; \& SILVA, A. C. 2011; Avaliação da sinergia entre a engenharia de processos e o processo de pensamento da teoria das restrições.. Prod. ILUS, TAB.Jun. 21(2),p. 284-300.

LAW, A.M.; \& KELTON, W.D. 2000. Simulation Modeling and Analysis. Boston: McGraw-Hill.

LEAL, F. 2003. Um diagnóstico do processo de atendimento a clientes em uma agência bancária através de mapeamento do processo e simulação computacional. 223p. Dissertação (Mestrado). Universidade Federal de Itajubá, UNIFEI, Itajubá.

MARTINS, J. L. F. A 2013. Avaliação da produtividade do processo de soldagem MAG por simulação com o método de hipercubo latino. Engevista volume: 15 issue:1 Pages: 104-117.

MENEZES, B. L. S.; DONATO, V.; SENNA, V.; SAMPAIO, R. R.; PASSOS, F. U. \& MIURA, M. N. Redução de custos nas organizações: complexidade e dinâmica das redes logísticas, 2012. PODES. Rio de Janeiro, , setembro a dezembro. v.4, n.3, p. 325-338.

MILARÉ, S. A.; \& YOSHIDA, E. M. P. Intervenção breve em organizações: mudança em coaching de executivos. Psicol. Estud. TAB. 2009 Dec.14(4): p. 717-727,

MOREIRA, D. A. 2007. Pesquisa operacional: curso introdutório. São Paulo: Thomson.

PASTORE, P.; GUIMARÃES A. M. C.; \& DIALLO M. 2010. Simulação computacional aplicada à logística de distribuição de uniformes da Marinha do Brasil. Rio de Janeiro. XXX ENEGEP, Out.

PEIXOTO, T. A., RANGEL, J. J. A., MATIAS, Í. O. M., MONTVECH, J. A. B., \& MIRANDA, R. C. 2013 URURAU.. Um ambiente para desenvolvimento de modelos de simulação a eventos discretos. Pesquisa Operacional para o Desenvolvimento v. 5, n. 3. P 373-405.

PEREIRA, C. R.; \& COSTA, M. A. B. Um modelo de simulação de sistemas aplicado à programação da produção de um frigorífico de peixe. 2012. Revista Produção Online, Florianópolis, SC, v.12, out./dez n. 4, p. 972-1001.

POROPUDAS, J.; \& VIRTANEN, K. 2011. Simulation metamodeling with dynamic Bayesian networks. European Journal of Operational Research 214. p644-655.

PRADO, D. S. 2010. Usando o Arena em simulação. Belo Horizonte: INDG Tecnologia e Serviços Ltda.

RANGEL, J.J.A.; SOUZA, A.A.; BASTOS, P.J.T.; \& BAPTISTA, R.C.T.2012. Simulação a eventos discretos para treinamento em sistemas de controle. PO Desenvolvimento. V.4. N. p 97-111.

SANTOS, P. M. R. dos; \& SANTOS M. I. R. dos. 2009. Using subsystem linear regression metamodels in stochastic simulation. European Journal of Operational Research 196. p1031-1040.

SARAIVA JÚNIOR, A. F.; TABOSA, C. de M.; \& COSTA, R. P. 2010.. Simulação de Monte Carlo aplicada à análise econômica de pedido. Revista Produção, 149-164, jan./mar v. 21, n. 1, p.. 149-161

SELLITTO, M. A.; \& WALTER, C. 2008. Medição e controle do tempo de atravessamento em um sistema de 
manufatura. Prod. GRA, ILUS,.TAB. Apr 15(1): p. 135-147,

SILVA, E. L.; \& MENEZES, E. M. 2001. Metodologia da pesquisa e elaboração de dissertação. Universidade Federal de Santa Catarina, Florianópolis.

VALENÇA, A. P. 2011. Simulação do fluxo de usuários do grupo de avaliação $e$ acompanhamento de pacientes especiais da Policlínica Naval Nossa Senhora da Glória. 150p. Dissertação (Mestrado). Universidade Federal do Rio de Janeiro, COPPE, Rio de Janeiro. 